\title{
Ein ideales Paar: Erstattungs- und Festbeträge zur Regulierung von Arzneimittelpreisen
}

CHRISTINA BODE,

ANTJE HAAS,

ANJA TEBINKA-OLBRICH

Christina Bode ist Referatsleiterin „ArzneimittelFestbeträge“ in der Abteilung Arznei- und Heilmittel beim GKV-Spitzenverband in Berlin

Dr. Antje Haas ist Leiterin der Abteilung Arznei- und Heilmittel beim GKV-

Spitzenverband

Dr. Anja Tebinka-Olbrich ist Referatsleiterin "AMNOG“" in der Abteilung Arzneiund Heilmittel beim GKVSpitzenverband in Berlin

Die Regulierung von Arzneimittelpreisen erfolgt seit 1989 über Arzneimittel-Festbeträge. Sie sind Erstattungshöchstgrenzen für Arzneimittel, zu denen die Krankenkassen die Kosten für verordnete Arzneimittel übernehmen und werden vom GKV-Spitzenverband für Gruppen vergleichbarer Arzneimittel festgesetzt. Als zusätzliches kollektivvertragliches Steuerungsinstrument wurden ab 2011 verhandelte Erstattungsbeträge für Arzneimittel mit neuen Wirkstoffen eingeführt, die nicht unmittelbar oder gar nicht festbetragsgeregelt werden können. In der aktuellen Auseinandersetzung über die grundsätzliche Ausrichtung beider Instrumente wird insbesondere von Industrieseite vorgetragen, dass beide Regelungsbereiche isoliert zu betrachten seien und „Arzneimittel mit Zusatznutzen“ nicht festbetragsgeregelt werden dürften. Dieser Beitrag zeigt, dass Festbeträge und Erstattungsbeträge nicht auf zwei getrennt zu betrachtende Marktsegmente gerichtet sind, sondern sich sowohl konzeptionell ergänzen als auch sequentiell anzuwenden sind.

\section{Erstattungsbeträge und Festbeträge: Gemeinsamkeiten und Unterschiede}

Mit der frühen Nutzenbewertung und der anschließenden Verhandlung von Erstattungsbeträgen hat der Gesetzgeber mit dem Arzneimittelmarktneuordnungsgesetz (AMNOG) vom 22.12.2010 ein weiteres, auf die Preise von Arzneimitteln gerichtetes Steuerungsinstrument eingeführt. Dieses erstreckt sich auf Arzneimittel mit neuen, patentgeschützten Wirkstoffen, die nicht unmittelbar oder gar nicht festbetragsgeregelt werden können. Dieser Bereich war lange Zeit ungeregelt. Als Folge öffnete sich die Schere zwischen einem vergleichsweise niedrigen Verordnungs- und einem überproportional hohen Umsatzanteil für nicht festbetragsgeregelte Arzneimittel immer weiter. Nach dem Scheitern einseitig festzusetzender Höchstbeträge (GKV-Wettbewerbsstärkungsgesetz vom 26.03.2007) sollen nun verhandelte Erstattungsbeträge für Arzneimittel mit neuen Wirkstoffen die Regelungslücke im festbetragsfreien Markt schließen. Wei- 
terhin wird damit ein Teil von Arzneimitteln mit patentgeschützten Wirkstoffen früher oder später festbetragsgeregelt. Nur bei nicht festbetragsfähigen Wirkstoffen (therapeutische Solisten) tritt der Erstattungsbetrag dauerhaft an die Stelle der früheren freien Preisbildung.

Dieser Logik folgend wurde die Festbetragsregelung mit dem AMNOG nicht verändert. Die Einführung der Festbeträge mit dem Gesundheits-Reformgesetz vom 20.12.1988 war mit der Erwartung einer umfassenden, nahezu 90-prozentigen Markdurchdringung durch Festbeträge verbunden (vgl. BT Drs. 11/2237 vom 03.05.1988, S. 275). Damit war von Beginn an klar, dass diese Regelung nicht etwa bevorzugt für die Phase nach dem Patentablauf vorgesehen, sondern insbesondere auch für patentgeschützte Wirkstoffe bestimmt ist. Von diesem Regelungsziel wurde mit dem AMNOG an keiner Stelle abgewichen. Im Gegenteil: Die Verzahnung von Erstattungsbeträgen mit Festbeträgen auch für Arzneimittel mit neuen Wirkstoffen zeigt sich darin, dass

- für Arzneimittel, die pharmakologisch-therapeutisch vergleichbar mit Festbetragsarzneimitteln sind, der medizinische Zusatznutzen als therapeutische Verbesserung entsprechend der Festbetragsregelung nachzuweisen ist ( $\mathbb{3} 35 \mathrm{a}$ Abs. 1 Satz 4 SGB V),

- bei nicht nachgewiesener therapeutischer Verbesserung eine unmittelbare Einordnung in eine bestehende Festbetragsgruppe erfolgt $(\mathbb{S} 35 \mathrm{a}$ Abs. 4 SGB V),

- die Verpflichtung zur Festsetzung eines Festbetrags oder Erstattungsbetrags unberührt bleibt, sofern bei nicht festgestelltem Zusatznutzen oder therapeutischer Verbesserung eine Bewertung des Nutzens unter Berücksichtigung von Kosten beauftragt wird ( $\$ 35$ Abs. 5a SGB V) und

- vereinbarte Erstattungsbeträge bei späterem Vorliegen der Voraussetzungen zur Bildung einer Festbetragsgruppe außerordentlich gekündigt werden können $(\mathbb{S} 130$ b Abs. 7 Satz 3 SGB V).

Somit werden Erstattungsbeträge nur dann verhandelt, wenn eine Einbeziehung in bereits bestehende Festbetragsgruppen nicht möglich ist. Folgerichtig können Erstattungsbeträge durch
Festbeträge abgelöst werden, sofern zu einem späteren Zeitpunkt eine Festbetragsgruppenbildung möglich ist. Das kann der Fall sein, wenn erst später weitere vergleichbare Wirkstoffe auf den Markt gebracht werden. In der zeitlichen Abfolge greift für prinzipiell festbetragsfähige Wirkstoffe also zunächst ein Erstattungsbetrag, bevor anschließend ein Festbetrag wirksam werden kann.

\subsection{Verfahren und Akteure}

Sowohl für Erstattungsbeträge als auch für Festbeträge gibt es klare gesetzliche Vorgaben. Beide Regelungen setzen zunächst eine entsprechende Beschlussfassung durch den Gemeinsamen Bundesausschuss (G-BA) voraus. Bei den Beschlüssen des G-BA zur Nutzenbewertung nach $\mathbb{S} 35$ a SGB V wurde zur Beschleunigung des Verfahrens - anders als bei den Beschlüssen zur Festbetragsgruppenbildung - auf einen Beanstandungsvorbehalt des Bundesministeriums für Gesundheit verzichtet $(\mathbb{S} 35$ a Abs. 3 Satz 6 SGB V).

Die Bewertung eines Zusatznutzens von Arzneimitteln mit neuen Wirkstoffen durch den $\mathrm{G}-\mathrm{BA}$ erfolgt gemäß $\mathbb{S} 35 \mathrm{a}$ SGB V innerhalb von sechs Monaten nach Inverkehrbringen. Sie basiert auf einem zum Zeitpunkt der Markteinführung vom pharmazeutischen Unternehmer einzureichenden Dossier, in dem auf Grundlage der Zulassungsunterlagen sowie weiterer Studien ein patientenrelevanter Zusatznutzen des Medikaments im Vergleich zu einer vom G-BA bestimmten zweckmäßigen Vergleichstherapie belegt werden kann. Auf dieser Grundlage stellt der G-BA fest, wie sich der Zusatznutzen eines Arzneimittels im Verhältnis zu der zweckmäßigen Vergleichstherapie darstellt. Unter bestimmten $\mathrm{Vo}^{-}$ raussetzungen legt der G-BA auch mehrere Alternativen für die zweckmäßige Vergleichstherapie nebeneinander fest. Aus diesen kann der pharmazeutische Unternehmer eine frei wählen.

In einem nachfolgenden Schritt wird dann der Erstattungsbetrag gemäß $\mathbb{S}$ 130b SGB V als Verhandlungsergebnis zwischen dem pharmazeutischen Unternehmer und dem GKV-Spitzenverband bestimmt. Können sich beide Verhand- lungspartner nicht binnen sechs Monaten einigen, folgt eine Schiedsentscheidung nach spätestens weiteren drei Monaten. Die Geltung des Erstattungsbetrags beginnt mit dem 13. Monat nach der Markteinführung.

Ebenso wie die Erstattungsbetragsregelung ist die Festbetragsregelung zweistufig angelegt. Zunächst bestimmt der G-BA gemäß $\mathbb{3} 35$ SGB V für welche Gruppen von Arzneimitteln Festbeträge festgesetzt werden können. Unterschieden werden drei Stufen von Festbetragsgruppen, die nicht in einem hierarchischen Verhältnis zueinander stehen $(\mathbb{S} 35$ Abs. 1 Satz 2 SGB V):

Stufe 1: Arzneimittel mit denselben Wirkstoffen

Stufe 2: Arzneimittel mit pharmakologisch-therapeutisch vergleichbaren Wirkstoffen, insbesondere mit chemisch verwandten Stoffen

Stufe 3: Arzneimittel mit therapeutisch vergleichbarer Wirkung, insbesondere Arzneimittelkombinationen

Nach dem Prozess der Gruppenbildung setzt der GKV-Spitzenverband für die durch den G-BA gebildeten Festbetragsgruppen nach den gesetzlichen Vorgaben des $\$ 35$ Abs. 5 SGB V jeweils einen Festbetrag fest. Aktuell entfallen im GKVArzneimittelmarkt fast 80 Prozent der Verordnungen und 40 Prozent des Umsatzes auf Arzneimittel mit Festbeträgen.

\section{Erstattungsbeträge können durch Festbeträge abgelöst werden, sofern zu einem späteren Zeitpunkt eine Festbetragsgruppenbildung möglich ist.}

Dies verdeutlicht den dauerhaften und maßgeblichen Beitrag der Festbeträge zu bezahlbaren Arzneimittelpreisen. Die Einsparungen durch dieses Instrument belaufen sich inzwischen auf jährlich 6,9 Milliarden Euro.

Arzneimittel mit neuen Wirkstoffen können allerdings häufig nicht unmittelbar nach ihrer Markteinführung festbetragsgeregelt werden. Das ist verfahrensbedingt: Die Bildung von Festbetragsgruppen der Stufe 1 (Arzneimittel 
mit identischem Wirkstoff) ist in der Regel erst nach Patentablauf und dann einsetzendem generischen Wettbewerb möglich. Eine Festbetragsgruppe der Stufe 2 oder 3 setzt die Verfügbarkeit weiterer Wirkstoffe voraus. Zudem besteht eine gesetzliche Ausnahme von der Festbetragsregelung für patentgeschützte Wirkstoffe mit neuartiger Wirkungsweise oder therapeutischer Verbesserung.

Während bei der Bewertung nach \35a SGB V dem Nutzen eines Arzneimittels im Vergleich zur zweckmäßigen Vergleichstherapie eine zentrale Bedeutung im Hinblick auf die Höhe des anschließend zu bestimmenden Erstattungsbetrags zukommt, setzt die Festbetragsregelung methodisch auf Preisunterschieden zwischen den in einer Festbetragsgruppe zusammengefassten und untereinander im Wettbewerb stehenden Arzneimitteln auf. Arzneimittel mit pharmakologisch-therapeutisch vergleichbaren Wirkstoffen können in gemeinsamen Gruppen der Stufe 2 zusammengefasst werden. Voraussetzung für eine auf pharmakologischer Ebene bestehende Vergleichbarkeit ist neben der Art und dem Aufbau einzelner Wirkstoffe ein vergleichbarer Wirkungsmechanismus. Als Aufgreifkriterium für eine mögliche Gruppenbildung der Stufe 2 ist die anatomisch-therapeutisch-chemischen Klassifikation der WHO (ATCCode) geeignet. Auf die pharmakologischen Eigenschaften kommt es hingegen bei einer Gruppenbildung der Stufe 3, bei der Wirkstoffe mit therapeutisch vergleichbarer Wirkung in gemeinsamen Festbetragsgruppen zusammengefasst werden, nicht an. Die therapeutische Vergleichbarkeit ergibt sich aus der arzneimittelrechtlichen Zulassung und ist bereits bei e i $\mathrm{n}$ e m gemeinsamen
Anwendungsgebiet aller Wirkstoffe gegeben (BSG-Urteil vom 01.03.2011, Az.: B 1 KR 13/10 R, Rn. 44). Über die therapeutische Vergleichbarkeit wird somit in einem ersten Schritt einer Festbetragsgruppenbildung ein gemeinsamer Bezugspunkt mehrerer Wirkstoffe hergestellt. Die therapeutische Vergleichbarkeit sagt nichts über den Nutzen eines Wirkstoffs im Vergleich zu einem anderen aus. Das Nutzenverhältnis zu den anderen Wirkstoffen derselben Festbetragsgruppe spielt erst bei der Beurteilung der therapeutischen Verbesserung eine zentrale Rolle, da ihre Bestätigung eine Festbetragsfreistellung durch NichtEinbeziehung bzw. Herausnahme aus einer Gruppe bewirkt.

\subsection{Erstattungshöhe und Preisgestaltung}

Die Höhe der Erstattungsbeträge soll aus bestehenden, patientenrelevanten Nutzenunterschieden neuer Arzneimittel im Vergleich zu bereits etablierten Therapien abgeleitet werden. Letztlich sollen die besser bewerteten Arzneimittel mit höheren Preisen belohnt werden, während der Erstattungsbetrag für Arzneimittel ohne nachgewiesenen Zusatznutzen nicht zu höheren Jahrestherapiekosten führen darf als die der zweckmäßigen Vergleichstherapie. Sind mehrere Alternativen für die zweckmäßige Vergleichstherapie durch den G-BA bestimmt, darf der Erstattungsbetrag für Arzneimittel ohne nachgewiesenen Zusatznutzen nicht zu höheren Jahrestherapiekosten führen als deren wirtschaftlichste Alternative. Die Preise der zweckmäßigen Vergleichstherapie dienen somit als Ausgangspunkt für die Erstattungsbeträge. Umso wichtiger ist, dass für die Monetarisierung des Zusatznutzens kein Algorithmus vorgeschrieben ist, sondern der Interessensausgleich im Verhandlungsprozess hergestellt wird.

Bei der Erstattungsbetragsregelung wird der vom pharmazeutischen Unternehmer verlangte Abgabepreis direkt durch den Erstattungsbetrag modifiziert. Dies erfolgt in Abhängigkeit vom Ausmaß und der Wahrscheinlichkeit des Zusatznutzens in a 11 e $\mathrm{n}$ zugelassenen Anwendungsgebieten gegenüber der zweckmäßigen Vergleichstherapie, wie sie vom G-BA festgelegt worden ist. Nur für das erste Jahr nach der Markteinführung wird weiterhin eine freie Preisbildung zugestanden. Die Preisfunktion des Erstattungsbetrags, die auch im Zusammenhang mit der Festsetzung von Festbeträgen sowie zur Kostendarstellung der zweckmäßigen Vergleichstherapie bedeutsam ist, wurde mit dem 14. SGB V-Änderungsgesetz vom 27.03.2014 klargestellt. Für den pharmazeutischen Unternehmer besteht ungeachtet dessen weiterhin die Möglichkeit, seinen Listenpreis frei festzusetzen und auszuweisen.

Im Unterschied zur Erstattungsbetragsregelung bleiben die pharmazeutischen Unternehmer bei Festbeträgen frei in ihrer Preisgestaltung. Als Erstattungshöchstgrenze berührt der Festbetrag den Abgabepreis des pharmazeutischen Unternehmers nicht direkt. Festbeträge sollen den gesetzlichen Vorgaben entsprechend einen wirksamen Preiswettbewerb auslösen und sich dazu an möglichst preisgünstigen Versorgungsmöglichkeiten ausrichten $(\mathbb{S} 35$ Abs. 5 Satz 2 SGB V). Die Höhe des jeweiligen Festbetrags ist ebenfalls gesetzlich bestimmt. Danach müssen mindestens 20 Prozent der Verordnungen und 20 Prozent der Packungen zum Festbetrag verfügbar sein

Abbildung 1: Festbetragsregelung zum 01.06.2014

\begin{tabular}{|c|c|c|c|c|c|c|c|c|}
\hline $\begin{array}{r}\text { Gruppierung } \\
\text { nach } \\
\S 35 \text { SGB V }\end{array}$ & \multicolumn{2}{|c|}{$\begin{array}{l}\text { Identische } \\
\text { Wirkstoffe }\end{array}$} & \multicolumn{2}{|c|}{$\begin{array}{c}\text { Pharmakologisch- } \\
\text { therapeutisch } \\
\text { vergleichbare Wirkstoffe }\end{array}$} & \multicolumn{2}{|c|}{$\begin{array}{c}\text { Therapeutisch } \\
\text { vergleichbare } \\
\text { Wirkung } \\
\text { (Stufe 3) }\end{array}$} & \multicolumn{2}{|c|}{$\begin{array}{c}\text { Gesamter } \\
\text { Festbetrags- } \\
\text { markt }\end{array}$} \\
\hline Festsetzung für & 300 & Gruppen & 66 & Gruppen & 58 & Gruppen & 424 & Gruppen \\
\hline mit & 192 & Wirkstoffen & 177 & Wirkstoffen & 144 & Wirkstoffkomb. & & \\
\hline Umsatz & 4,7 & Mrd. $€$ & 5,6 & Mrd. $€$ & 2,1 & Mrd. $€$ & 12,4 & Mrd. $€$ \\
\hline Verordnungen & 221,4 & Mio. & 223,7 & Mio. & 61,4 & Mio. & 506,5 & Mio. \\
\hline Packungen & 15.181 & Packungen & 12.271 & Packungen & 4.904 & Packungen & 32.356 & Packungen \\
\hline
\end{tabular}


( $\int 35$ Abs. 5 Satz 5 SGB V). Ziel dieser Regelung ist es, Ärzte und Patienten zu motivieren, preisgünstige Arzneimittel mit vergleichbarer Wirkung zu wählen. Zugleich erhalten pharmazeutische Unternehmen einen Anreiz, ihre Preise an den Festbeträgen auszurichten, damit ihre Produkte weiterhin verordnet werden. Auf diese Weise fördern Festbeträge den Wettbewerb um niedrige Arzneimittelpreise, ohne die therapeutisch notwendige Arzneimittelauswahl einzuschränken. Darüber hinaus kann der GKV-Spitzenverband besonders preisgünstige Festbetragsarzneimittel von der gesetzlichen Zuzahlung freistellen, wenn daraus weitere Einsparungen zu erwarten sind ( $\$ 31$ Abs. 3 Satz 4 SGB V).

\subsection{Zusatznutzen und Therapeutische Verbesserung}

Bei der Nutzenbewertung gemäß $\mathbb{3} 3 \mathrm{a}$ SGB V wird die Wahrscheinlichkeit (Anhaltspunkt, Hinweis, Beleg) und das Ausmaß (geringerer, keiner, nicht quantifizierbar, gering, beträchtlich, erheblich) eines Zusatznutzens, ggf. differenziert nach den verschiedenen Anwendungsgebieten und Patientengruppen eines Arzneimittels, wirkstoffspezifisch bewertet ( $\int 5$ AM-NutzenV). Auf dieser Grundlage wird anschließend ein diese Differenzierungen berücksichtigender Erstattungsbetrag verhandelt.

\section{Abbildung 2: Ausmaß und Wahrscheinlichkeit des Zusatznutzens}

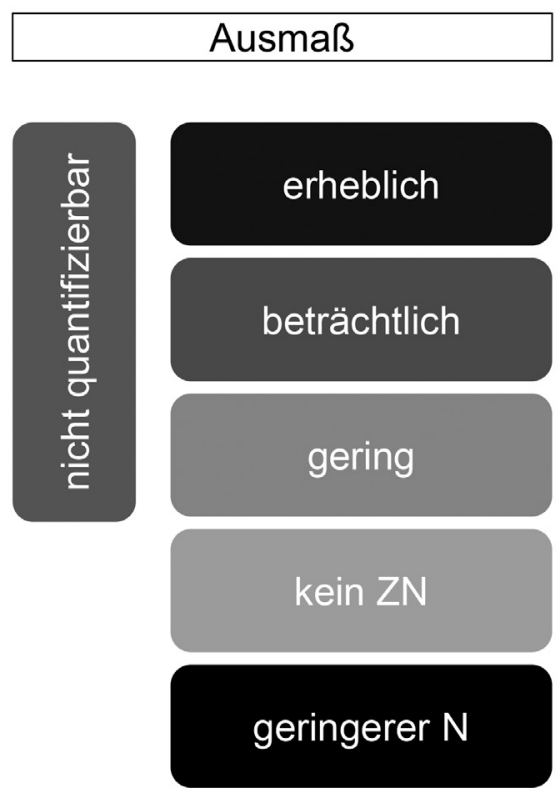

Quelle: Eigene Darstellung
Augenfällig ist in diesem Zusammenhang eine häufig undifferenzierte Verwendung des Begriffs „Zusatznutzen“ in der Fach-Öffentlichkeit. So wird auch dann von „Arzneimitteln mit Zusatznutzen“ gesprochen, wenn dieser (noch) nicht belegt ist, sondern es nur einen Hinweis bzw. Anhaltspunkt gibt oder wenn lediglich für eine Minderheit der von der Zulassung umfassten Patientengruppen ein Zusatznutzen belegt ist. In der öffentlichen Darstellung resultieren aus den differenzierten Vorgaben zur Bewertung eines Zusatznutzens jedoch Schlagzeilen wie „Frühe Nutzenbewertung: Drei neue Arzneimittel mit Zusatznutzen“ (DAZ online 02.05.2013). Im Ergebnis hatte der G-BA bei zwei Arzneimitteln „Anhaltspunkte“ für einen geringen bzw. beträchtlichen Zusatznutzen in jeweils einem Anwendungsgebiet festgestellt. Bei dem dritten Arzneimittel wurde lediglich ein geringer Zusatznutzen festgestellt. Dass derartige Nutzenbewertungsergebnisse einer Festbetragsgruppenbildung nicht grundsätzlich entgegenstehen, liegt auf der Hand.

Im Rahmen der Festbetragsregelung kommt es auf etwaig bestehende Nutzenunterschiede zwischen unterschiedlichen Wirkstoffen nicht an, sofern sie nicht das Ausmaß einer therapeutischen Verbesserung annehmen. Was unter einer therapeutischen Verbesserung zu verstehen ist, hat der Gesetzgeber durch den mit dem Gesetz zur Verbesserung der Wirtschaftlichkeit in der Arzneimittelversorgung

\section{Wahrscheinlichkeit}

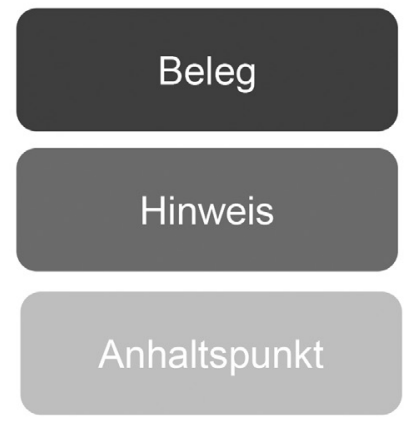
aufgenommenen $\mathbb{3} 35$ Abs. 1b SGB V konkretisiert. Danach liegt eine therapeutische Verbesserung vor, wenn das Arzneimittel einen therapierelevanten höheren Nutzen als andere Arzneimittel dieser Wirkstoffgruppe hat und deshalb als zweckmäßige Therapie regelmäßig oder auch für relevante Patientengruppen oder Indikationsbereiche den anderen Arzneimitteln dieser Gruppe vorzuziehen ist. Die Bewertung einer therapeutischen Verbesserung erfolgt ausschließlich in den gemeinsamen Anwendungsgebieten einer Festbetragsgruppe.

Die gesetzliche Formulierung zeigt, dass für die Bestätigung einer therapeutischen Verbesserung eine Erheblichkeitsschwelle besteht, die sich nicht per se aus einem höheren Nutzen ableitet. Dieser höhere Nutzen muss vielmehr so umfassend sein, dass deshalb das Arzneimittel als zweckmäßige Therapie regelmäßig vorzuziehen ist $(\mathbb{S} 35$ Abs. 1b Satz 1 SGB V). Dies bedeutet, dass ein Wirkstoff einen so hohen zusätzlichen Nutzen haben müsste, dass der verordnende Arzt bei gegebener Diagnose gleichsam einen Behandlungsfehler begehen würde, wenn er nicht den betroffenen Wirkstoff, sondern ein anderes Arzneimittel der Festbetragsgruppe verordnet.

Im Hinblick auf Nutzenunterschiede, die eine therapeutische Verbesserung nicht begründen können, bedeutet dies, dass die jeweiligen Wirkstoffe als gleichwertige Versorgungsalternativen in einer Festbetragsgruppe zusammengefasst werden können und auf dieser Grundlage ein einheitlicher Festbetrag festgesetzt werden kann (BSG-Urteil vom 01.03.2011, Az.: B 1 KR 13/10 R, Rn. 20).

Während es bei der frühen Nutzenbewertung nach $\mathbb{S} 35$ a SGB V um die differenzierte Feststellung der Wahrscheinlichkeit und des Ausmaßes des Nutzens im Vergleich zur zweckmäßigen Vergleichstherapie geht, ist der Bewertungsfokus bei der Festbetragsregelung anders ausgerichtet. Hier spielt der Nutzen im Vergleich zu anderen Arzneimitteln einer Festbetragsgruppe zum Nachweis einer therapeutischen Verbesserung eine zentrale Rolle. Dementsprechend werden bei der frühen Nutzenbewertung neue, mit Festbetragsarzneimitteln pharmakologisch-therapeutisch vergleichbare Arzneimittel unmittelbar im Festbetragszusammenhang bewertet $(\mathbb{S} 35 \mathrm{a}$ Abs. 1 
Satz 4 SGB V). Das bedeutet, dass der Zusatznutzen nicht in differenzierten Abstufungen und Wahrscheinlichkeiten dargestellt wird, sondern ausschließlich als Ja-Nein-Entscheidung bewertet wird, ob eine therapeutische Verbesserung im Sinne der Festbetragsregelung belegt ist oder nicht. Bloße Anhaltspunkte oder Hinweise sind somit von vornherein nicht zum Nachweis einer therapeutischen Verbesserung geeignet. Kann für festbetragsfähige Wirkstoffe der Zusatznutzen nach $\mathbb{S} 35$ a SGB V nicht als therapeutische Verbesserung entsprechend \35 Abs. 1b Satz 1 bis 5 SGB V nachgewiesen werden, werden die Wirkstoffe unmittelbar der bestehenden Festbetragsgruppe zugeordnet. Diesen gesetzlichen Vorgaben trägt der G-BA durch eine separate Dossier-Vorlage für mit Festbetragsarzneimitteln pharmakologischtherapeutisch vergleichbare Arzneimittel Rechnung (Anlage VI zum 5. Kapitel der Verfahrensordnung des G-BA).

\subsection{Bewertungszeitpunkt und Studienlage}

Mit der Nutzenbewertung nach $\mathbb{S} 35 \mathrm{a}$ SGB V soll der therapeutische Stellenwert eines Arzneimittels mit einem neuen Wirkstoff bereits frühzeitig gegenüber einer zweckmäßigen Vergleichstherapie bestimmt werden. Dabei sind die Wahrscheinlichkeit und das Ausmaß eines Zusatznutzens darzustellen, ggf. differenziert nach verschiedenen Anwendungsgebieten. Der frühe Bewertungszeitpunkt bedingt, dass

- die Bewertung grundsätzlich auf Basis von Zulassungsstudien vorgenommen wird ( $\mathbb{5}$ Abs. 3 AM-NutzenV)

- sofern zum Zeitpunkt der Bewertung valide Daten zu patientenrelevanten Endpunkten noch nicht vorliegen können, die Bewertung auf der Grundlage der verfügbaren Evidenz unter Berücksichtigung der Studienqualität mit Angabe der Wahrscheinlichkeit für den Beleg eines Zusatznutzens erfolgt $(\mathbb{S}$ Abs. 5 AM-NutzenV)

- Zusatznutzennachweise auf der Basis indirekter Vergleiche zulässig sind ( $\mathbb{S}$ 5 Abs. 5 AM-NutzenV).

Zulassungsstudien dienen dazu, die pharmazeutische Qualität, Wirksamkeit und Unbedenklichkeit als notwendige Voraussetzung für eine Zulassung zu belegen. Die arzneimittelrechtliche $\mathrm{Zu}-$ lassung stellt also eine produktbezogene Einzelfallentscheidung dar. Dahingegen ist der Nutzen eines Arzneimittels, dessen Feststellung eine vergleichende Bewertung voraussetzt, nicht Gegenstand der Zulassung. Bei der frühen Nutzenbewertung ist die Erkenntnislage zu den gesetzlich bestimmten patientenrelevanten Endpunkten (insbesondere Morbidität) eines Arzneimittels im Vergleich zu anderen Therapien zunächst häufig eher gering. Es kann also eine Diskrepanz bestehen zwischen der Aussagekraft der für die Zulassung durchgeführten Studien einerseits und den in der Praxis auftretenden Anforderungen an ein Arzneimittel andererseits, insbesondere beim Fehlen klinischer Studien zu patientenrelevanten Endpunkten (vgl. BSG-Urteil vom 01.03.2011, Az.: B 1 KR 13/10 R, Rn. 34). Dies ist auch darin begründet, dass vergleichende Studien gegenüber einer anderen Therapie häufig noch nicht vorliegen oder in den Zulassungsstudien nicht auf patientenrelevante Endpunkte, sondern auf Surrogatparameter, die einen Nutzen für Patienten nicht endgültig belegen können, abgestellt wird. Daraus resultiert auch, dass der Nutzen in einem frühen Stadium nach Markteinführung eher überschätzt, die Risiken aber unterschätzt werden. Die zum Zulassungszeitpunkt häufig noch unsichere Erkenntnislage ist aber Grundlage für die frühe Nutzenbewertung nach $\$ 35$ a SGB V.

Dass sich der Wissensstand nach der Zulassung auf Basis weiterer Studien und Analysen ändert, ist somit ganz normal

\section{Die frühe Nutzenbewertung darf kein einmaliger Vorgang zu einem Wirkstoff bleiben, sondern muss in einen zyklischen Prozess münden.}

und kommt beispielsweise darin zum Ausdruck, dass Fachinformationen zugelassener Arzneimittel nachträglich um Warnhinweise ergänzt werden. Beispielhaft sei an dieser Stelle der Wirkstoff Ponatinib genannt. Bereits vier Monate nach der Markteinführung in Deutschland wurde in einem Rote-Hand-Brief vor schweren Nebenwirkungen gewarnt (Gefäßverschlüsse mit Herzinfarkten, Thrombosen). Mit Rote-Hand-Briefen werden die Fachkreise über neu erkannte, bedeutende Arzneimittelrisiken und Maßnahmen zu ihrer Minderung informiert. Diese Nebenwirkungen führten in den USA sogar dazu, dass das Arzneimittel zeitweise vom Markt genommen werden musste.

Somit sind zum Zeitpunkt der Markteinführung nach der Zulassung weder alle wesentlichen Nebenwirkungen noch alle Potentiale eines Arzneimittels bekannt. Umso wichtiger ist in diesem Zusammenhang, dass die frühe Nutzenbewertung kein einmaliger Vorgang zu einem Wirkstoff bleibt, sondern in einen zyklischen Prozess anhand neuer Daten zu patientenrelevanten Endpunkten aktualisierter Zusatznutzenbewertungen und daran adjustierter Preisverhandlungen mündet.

Im Rahmen der frühen Nutzenbewertung nach $\mathbb{S} 35$ a SGB V werden neue, mit Festbetragsarzneimitteln pharmakologisch-therapeutisch vergleichbare Medikamente unmittelbar im Festbetragszusammenhang bewertet. Dabei ist vorrangig auf direkte Vergleichsstudien mit anderen Arzneimitteln der jeweiligen Festbetragsgruppe abzustellen $(\mathbb{S} 5$ Abs. 2 AM-NutzenV). Die herausragende Bedeutung direkt vergleichender Studien für die Festbetragsregelung hat der Gesetzgeber bereits durch $\mathbb{} 35$ Abs. 1b Satz 5 SGB V zum Ausdruck gebracht. Sofern bereits Festbetragsgruppen mit pharmakologisch-vergleichbaren Wirkstoffen (Stufe 2) bestehen, kann sich ein pharmazeutischer Unternehmer frühzeitig auf diese Anforderung einstellen. Dasselbe gilt auch für den Fall, dass es zwar noch keine Festbetragsgruppe, wohl aber entsprechende, pharmakologisch-therapeutisch vergleichbare Wirkstoffe derselben Wirkstoffklasse gibt. Damit fördert die Festbetragsregelung die Konzentration auf patientenrelevante Forschung und Entwicklung, insbesondere auch nach der Markteinführung neuer Arzneimittel.

Für nicht unmittelbar festbetragsfähige Arzneimittel spiegelt die Nutzenbewertung nach $\mathbb{3} 35$ a SGB V den Nutzen zu einem frühen Zeitpunkt in allen Anwendungsgebieten der Zulassung gegenüber jeweils einer zweckmäßigen Vergleichstherapie wider. Nicht mehr und nicht weniger. Damit wird jedoch nicht der Nutzen zwingend vollständig 
(z.B. auch gegen andere zweckmäßige Vergleichstherapien) und für alle Zeiten zutreffend abgebildet (ansonsten wäre eine spätere Nutzenbewertung entbehrlich). Das mag auch ein Grund dafür sein, dass bisher nur sehr wenige Belege für einen beträchtlichen und noch gar kein Beleg für einen erheblichen Zusatznutzen festgestellt werden konnten.

Im Rahmen der Festbetragsregelung ist eine therapeutische Verbesserung in den gemeinsamen Anwendungsgebieten gegenüber anderen Arzneimitteln einer Festbetragsgruppe nachzuweisen $(\mathbb{S} 35$ Abs. 1b Sätze 1 und 2 SGB V). Bezugspunkt für die Anerkennung einer therapeutischen Verbesserung können nur die gemeinsamen Anwendungsgebiete einer Festbetragsgruppe sein. Dies ergibt sich bereits daraus, dass die Feststellung der therapeutischen Vergleichbarkeit voraussetzt, dass die Arzneimittel für ein oder mehrere gemeinsame Anwendungsgebiete arzneimittelrechtlich zugelassen sind (BT Drs. 16/194 vom 13.12.2005, S. 8).

Der Nachweis einer therapeutischen Verbesserung erfolgt auf Basis der Fachinformationen und klinischer Studien. Hier sind vorrangig direkte Vergleichsstudien mit anderen Arzneimitteln derselben Gruppe mit patientenrelevanten Endpunkten, insbesondere Mortalität, Morbidität und Lebensqualität, zu berücksichtigen $(\mathbb{S} 35 \mathrm{Abs}$. 1b Sätze 4 und 5 SGB V). Erforderlich ist dabei der Nachweis der therapeutischen Verbesserung in einer für die sichere Beurteilung ausreichenden Zahl von Behandlungsfällen auf der Grundlage wissenschaftlich einwandfrei geführter Statistiken über die Zahl der behandelten Fälle und die Therapierelevanz. Die höchste Beweiskraft haben danach direkte Vergleichsstudien mit anderen Wirkstoffen derselben Festbetragsgruppe. Nur soweit derartige Untersuchungen nicht existieren, kann im Einzelfall auf andere, hinreichend aussage- und beweiskräftige Studien ausgewichen werden. Sie müssen in jedem Fall mit dem Primärziel des Erreichens patientenrelevanter Endpunkte durchgeführt worden sein. Studien, die als Primärziel lediglich Surrogatparameter formuliert haben, kommen dagegen zum Nachweis einer therapeutischen Verbesserung nicht in Betracht (BSG-Urteil vom 01.03.2011, Az.: B 1 KR 13/10 R, Rn. 54).

Zusammenfassend ist der Festbetragsund der Erstattungsbetragsregelung gemeinsam, dass die Höhe der Erstattung durch die Krankenkassen auf den medizinischen Nutzen ausgerichtet ist. Von der Festbetragsregelung werden Arzneimittel mit nachgewiesener therapeutischer Verbesserung gemäß $\mathbb{S} 35$ Absatz 1b Satz 1 bis 5 SGB V quasi als „Belohnung“ eines besonders hohen patientenrelevanten Nutzens im gemeinsamen Anwendungsgebiet freigestellt. Bei den Erstattungsbeträgen modifiziert sich der Abgabepreis in Abhängigkeit von Ausmaß und Wahrscheinlichkeit des Zusatznutzens in allen zugelassenen Anwendungsgebieten gegenüber der zweckmäßigen Vergleichstherapie, wie sie vom G-BA festgelegt worden ist.

\section{Die Potentiale beider Steuerungsinstrumente}

Während die frühe Nutzenbewertung und die anschließend zu verhandelnden Erstattungsbeträge auf nicht bzw. nicht unmittelbar festbetragsfähige Arzneimittel ausgerichtet sind, sind Festbeträge weiterhin das vorrangige Regulierungsinstrument für wirkstoffidentische Arzneimittel (Stufe 1) sowie für in ihrem Wirkmechanismus und hinsichtlich ihrer Wirkung vergleichbare Wirkstoffe (Stufen 2 und 3).

\subsection{Vor- und Nachteile von Erstattungsbeträgen}

Der entscheidende Vorteil der ab 2011 eingeführten Erstattungsbeträge ist darin zu sehen, dass das Preismonopol der pharmazeutischen Unternehmer zugunsten zusatznutzenorientierter Preismodifikation nach einem Jahr freier Vermarktungsmöglichkeiten gebrochen wird. Erstattungsbeträge sind insbesondere im Hinblick auf das Marktsegment nicht festbetragsfähiger Arzneimittel von zentraler Bedeutung, da es hierfür bisher keinen anderen Steuerungsmechanismus gab. Wichtig sind sie zudem als „Übergangsregelung“ für grundsätzlich festbetragsfähige Arzneimittel, sofern auf Grund weiterer Marktveränderungen (Einführung im Wettbewerb stehender weiterer Wirkstoffe) erst zu einem späteren Zeitpunkt eine Festbetragsgruppenbildung möglich wird.

Auch die regelhafte Nutzenbewertung von Arzneimitteln mit neuen Wirkstoffen bereits zum Zeitpunkt des Inverkehr- bringens ist von unschätzbarem Wert. Sie schafft praktisch das bisherige Informationsmonopol der pharmazeutischen Industrie über die Vorzüge und Nachteile neuer Arzneimittel ab. Schon mit der 
entscheidung des AMNOG ist die Preisfreiheit und uneingeschränkte Erstattungsfähigkeit im gesamten ersten Jahr nach dem Inverkehrbringen, sofern eine unmittelbare Einbeziehung in die Festbetragsregelung nicht möglich ist. Über einen hohen Einstiegspreis kann damit die Einsparwirkung der Erstattungsbeträge zumindest teilkompensiert werden. Darüber hinaus etabliert sich im Wege der internationalen Preisreferenzierung ein hohes europäisches Preisniveau, welches dann wiederum in den Erstattungsbetragsverhandlungen in Deutschland zu berücksichtigen ist. Wenn Deutschland zudem weiterhin von einer Entscheidung zur Erstattungsfähigkeit absieht, sollte konsequenterweise der Erstattungsbetrag insbesondere bei fehlendem Zusatznutzen unmittelbar ab dem ersten Tag des InVerkehr-Bringens gelten.

\subsection{Möglichkeiten und Grenzen von Festbeträgen}

Mit dem AMNOG war gemäß $\mathbb{3} 35 \mathrm{a}$ Abs. 6 SGB V zunächst eine Prüfung des Bestandsmarktes vorgesehen. Danach sollte der G-BA auch für bereits im Verkehr befindliche Arzneimittel eine Nutzenbewertung veranlassen können. Anschließend sollten Erstattungsbeträge verhandelt werden. Mit dem 14. SGB V-Änderungsgesetz vom 27.03.2014 wurde diese Regelung jedoch wieder aufgehoben, so dass ein Marktsegment von großer wirtschaftlicher und versorgungspolitischer Bedeutung zunächst preislich ungeregelt bleibt. Der Wegfall der Bestandsmarktprüfung bestätigt somit die Bedeutung von Festbeträgen, da hier auf Erstattungsbeträge als zumindest vorübergehende, ausgaben- und vor allem qualitätssteuernde Regelung verzichtet wurde.

Der Zeitraum, bis zu dem eine Festbetragsgruppe für neue Wirkstoffe, die nicht unmittelbar in die Festbetragsregelung einbezogen werden, gebildet werden kann, kann jedoch sehr lang sein: Gemäß \ 35 Abs. 1 SGB V dürfen nämlich für Arzneimittel mit patentgeschützten Wirkstoffen, deren Wirkungsweise neuartig ist, keine Festbeträge festgesetzt werden. Eine neuartige Wirkungsweise ist gegeben, solange ein Wirkstoff, der als erster einer Wirkstoffgruppe in Verkehr gebracht worden ist, unter Patentschutz steht $(\mathbb{S}$ 35 Abs. 1 Satz 4 SGB V). Festbeträge können somit erst frühestens nach $\mathrm{Pa}$ - tentablauf des ersten Wirkstoffs mit derselben Wirkungsweise gebildet werden. Der Zeitraum zwischen der Einführung eines neuen Wirkstoffs und dem Patentablauf beträgt in der Regel über zehn Jahre.

Auf die Neuartigkeit der Wirkungsweise kommt es hingegen bei einer Gruppenbildung auf der Grundlage des $\mathbb{3} 35$ Abs. 1a SGB V (Festbetragsgruppen mit ausschließlich patentgeschützten Wirkstoffen) nicht an. Solche Gruppen sind jedoch erst von dem Zeitpunkt an möglich, an dem mindestens drei vergleichbare Wirkstoffe verfügbar sind. Eine Gruppenbildung auf dieser Basis wäre angesichts der Vergleichbarkeit der Wirkstoffe Linagliptin, Saxagliptin, Sitagliptin und Vildagliptin denkbar, die alle einer Nutzenbewertung unterzogen wurden. Gleichwohl ist Grundvoraussetzung für jede Festbetragsgruppenbildung, dass durch die Festbeträge weitere Wirtschaftlichkeitspotentiale erschlossen werden. Dies ist derzeit für die genannten Wirkstoffe nicht der Fall. Darüber hinaus sind inzwischen zwei der Wirkstoffe vom Markt genommen worden, so dass die Grundvoraussetzung zur Gruppenbildung auf der Grundlage des $₫ 35$ Abs. 1a SGB V (mindestens drei vergleichbare Wirkstoffe) ohnehin nicht mehr erfüllt ist.

Die Nutzenbewertung nach $\$ 35$ a SGB $\mathrm{V}$ hat den Vorteil, dass zum Zeitpunkt der Markteinführung eine transparente Studienlage vorliegt. Das kann zukünftig die Bildung von Festbetragsgruppen insofern befördern, da frühzeitig erkennbar wird, ob Wirkstoffe das Potential für eine therapeutische Verbesserung haben. Am Beispiel der o.g. ADHSWirkstoffe hat die frühe Nutzenbewertung gezeigt, dass der neuere Wirkstoff im Vergleich zu dem älteren keinen Zusatznutzen hat, insofern also auch kein Potential für eine therapeutische Verbesserung bestehen kann. Gleichwohl ist es nicht möglich, jetzt unmittelbar beide Wirkstoffe in einer Festbetragsgruppe zusammenzufassen, da eine Festbetrags-

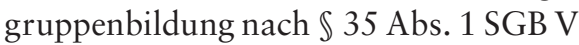
frühestens nach Patentablauf des ersten Wirkstoffs erfolgen kann.

Mit Apixaban ist seit Juni 2011 ein Wirkstoff zur Blutgerinnungshemmung auf dem Markt, für den im Rahmen der frühen Nutzenbewertung lediglich bei einem Teil der Zulassungspopulation ein
Hinweis einen geringen Zusatznutzen festgestellt werden konnte. Der Wirkstoff kann möglicherweise in Zukunft mit den beiden bereits verfügbaren und ebenfalls noch patentgeschützten Wirkstoffen Dabigatran und Rivaroxaban in einer gemeinsamen Festbetragsgruppe zusammengefasst werden. Allerdings zeichnet sich bereits ab, dass eine wirtschaftliche Gruppenbildung auf Basis des $\mathbb{3} 35$ Abs. 1a SGB V (Gruppen für ausschließlich patentgeschützte Wirkstoffe) derzeit nicht möglich ist. Alternativ kommt eine Gruppenbildung nach $\mathbb{} 35$ Abs. 1 SGB V in Frage, die allerdings erst nach dem Patentablauf des ersten Wirkstoffs realisiert werden kann.

\section{Diskussion und Fazit}

Von Seiten der pharmazeutischen Industrie wird inzwischen gefordert, „Arzneimittel mit Zusatznutzen“ bis zum Patentablauf von der Festbetragsregelung auszunehmen. Dabei bleibt offensichtlich bewusst unklar, was unter einem „Arzneimittel mit Zusatznutzen“ überhaupt zu verstehen ist: Hat ein Arzneimittel bereits einen Zusatznutzen, wenn lediglich Anhaltspunkte oder Hinweise auf einen möglichen Zusatznutzen bestehen, dieser jedoch nicht belegt ist? Was ist, wenn lediglich für eine sehr kleine Patientengruppe ein Zusatznutzen belegt ist, für die maßgeblichen Anwendungsgebiete eines Arzneimittels jedoch nicht? Hier sei als Beispiel der Beschluss des G-BA

\section{Die Forderung der Industrie hätte zur Folge, dass die Bildung neuer Festbetragsgruppen erschwert bzw. völlig unmöglich würde.}

für den Wirkstoff Axitinib vom März 2013 angeführt, bei dem für lediglich ein Prozent der Patientenpopulation ein Hinweis auf einen geringen Zusatznutzen festgestellt worden war.

Möglicherweise resultieren derartige Forderungen aus einem durchaus verbreiteten Missverständnis, wonach Festbeträge nur dann zur Anwendung kommen sollen, wenn kein Zusatznutzen besteht. In $\mathbb{3} 35 \mathrm{a}$ SGB V ist geregelt, dass für Arzneimittel, die pharmakologischtherapeutisch vergleichbar mit Festbe- 
tragsarzneimitteln sind, der Zusatznutzen als therapeutische Verbesserung nachzuweisen ist. Gelingt der Nachweis nicht, kann das Arzneimittel unmittelbar der Festbetragsregelung zugeführt werden $(\mathbb{S} 35$ a Abs. 1 Sätze 4 und 5 sowie Abs. 4 SGB V). Das heißt, die Einbeziehung in eine bestehende Festbetragsgruppe kommt immer dann in Frage, wenn kein Zusatznutzen im Sinne einer therapeutischen Verbesserung besteht. Bei der Bewertung des Zusatznutzens als therapeutische Verbesserung kommt es auf mögliche Nutzendimensionen nur dann an, wenn sie die Erheblichkeit einer therapeutischen Verbesserung erreichen.

Für alle anderen Arzneimittel, die nicht pharmakologisch-therapeutisch vergleichbar mit Festbetragsarzneimitteln sind, wird stets eine ,reguläre“ Nutzenbewertung durchgeführt, bei der das Ausmaß und die Wahrscheinlichkeit eines Zusatznutzens differenziert bestimmt werden. Bei einer möglicherweise später folgenden Festbetragsgruppenbildung kommt es bezüglich des Nachweises einer therapeutischen Verbesserung ausschließlich darauf an, ob im gemeinsamen Anwendungsgebiet der Festbetragsgruppe Belege zum Nachweis einer therapeutischen Verbesserung vorliegen oder nicht. Dieses Vorgehen steht im Einklang mit der Nutzenbewertung für bereits bei Markteinführung mit Festbetragsarzneimitteln vergleichbare Arzneimittel, bei denen der Zusatznutzen sofort als therapeutische Verbesserung nachzuweisen ist.

Im Rahmen einer Festbetragsgruppenbildung sind möglicherweise vorliegende Nutzenbewertungen nach $\$ 35$ a SGB V daraufhin zu prüfen, ob sie geeignet sind, eine Ausnahme von der Festbetragsregelung zu begründen. Selbst wenn jedoch im Rahmen der frühen Nutzenbewertung für nicht unmittelbar festbetragsfähige Arzneimittel ein bedeutsamer Zusatznutzen festgestellt werden kann, heißt dies noch wenig: So wurde für den Wirkstoff Vemurafenib zur Behandlung des metastasierten und lokal inoperablen Hautkrebses mit einer speziellen Biomarker-Positivität ein Hinweis auf einen beträchtlichen Zusatznutzen festgestellt, während für den vergleichbaren Wirkstoff Dabrafenib kein Zusatznutzen festgestellt werden konnte. Das hat nichts damit zu tun, dass Dabrafenib schlechter ist als Vemurafenib. Das Ergebnis resultiert lediglich daraus, dass der erste Wirkstoff Vemurafenib anders als der nachfolgende mit dem Wirkstoff Dacarbazin verglichen wurde und in der Folge dann selbst zweckmäßige Vergleichstherapie für die "Nachfolger" im selben Anwendungsgebiet wurde. Insofern ist eine generelle Kategorisierung eines im Rahmen der frühen Nutzenbewertung festgestellten Zusatznutzens nicht geeignet, um eine Ausnahmeregelung von der Festbetragsregelung über die therapeutische Verbesserung begründen zu können.

Worum es bei den industrieseitig vorgetragenen Forderungen nach einer Sonderregelung für „Arzneimittel mit Zusatznutzen" aber letztlich geht, ist etwas anderes: Das mit dem AMNOG vorgesehene Miteinander von Festbeträgen und Erstattungsbeträgen soll durch einen Vorrang der Erstattungsbeträge abgelöst werden. Indem dann jede Art eines Zusatznutzens in welchem Anwendungsgebiet auch immer zur Festbetragsfreiheit führt, soll die Bildung neuer Festbetragsgruppen erschwert bzw. völlig unmöglich gemacht werden.

Letztlich kommt hier erneut der altbekannte Wunsch der Industrie zum Ausdruck, patentgeschützte Arzneimittel von der Festbetragsregelung auszunehmen. Mit dieser Forderung konnte sich die pharmazeutische Industrie schon einmal, allerdings nur vorübergehend, durchsetzen, als mit dem 7. SGB V-Änderungsgesetz vom 28.10.1996 neue Arzneimittel mit patentgeschützten Wirkstoffen von der Festbetragsregelung ausgenommen wurden. In der Folge sind jedoch die Preise für diese Arzneimittel so massiv gestiegen, dass die Regelung mit dem GKV-Modernisierungsgesetz (GMG) vom 14.11.2003 wieder rückgängig gemacht wurde. Zugleich wurde mit dem GMG die Möglichkeit der Gruppenbildung für ausschließlich patentgeschützte Wirkstoffe geschaffen ( $\mathbb{S} 35$ Abs. 1a SGB V). Ausdrücklich sollten dadurch aus dem Preiswettbewerb zwischen patentgeschützten Arzneimitteln der gleichen Wirkstoffklasse resultierende Wirtschaftlichkeitsreserven erschlossen werden. Nur patentgeschützte Arzneimittel mit neuartigen Wirkstoffen einer neuen Wirkstoffklasse, für die es keine vergleichbaren Analog-Arzneimittel gibt, oder patentgeschützte Arzneimittel mit therapeutischer Verbesserung sollten von der Festbetragsbildung ausgenommen bleiben, um den Anreiz zur Entwicklung innovativer Arzneimittel zu erhalten (BTDrs. 15/1525 vom 08.09.2003, S. 87).
Zusammenfassend weist das AMNOG den richten Weg zu einer seit Langem erforderlichen Regulierung für Arzneimittel mit neuen Wirkstoffen. Dabei spielen sowohl Erstattungsbeträge als auch Festbeträge eine wesentliche Rolle. Während Erstattungsbeträge bereits frühzeitig nach dem Inverkehrbringen von Arzneimitteln mit neuen Wirkstoffen greifen, ist die Bildung neuer Festbetragsgruppen häufig erst zu einem späteren Zeitpunkt möglich. Somit steht außer Frage, dass sich beide Instrumente in einem ständiger Veränderung unterworfenen Arzneimittelmarkt sinnvoll ergänzen.

\section{Qualitätsverbesserung} als Mittel der gesundheitspolitischen Steuerung

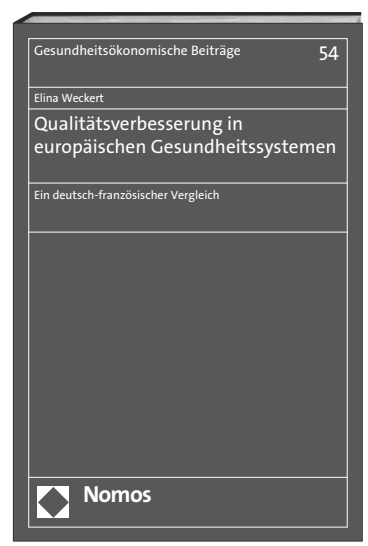

\section{Qualitätsverbesserung in europäischen Gesundheitssystemen}

Ein deutsch-französischer Vergleich

Von Elina Weckert

2014, 399 S., brosch., 88,-€

ISBN 978-3-8487-1270-0

(Gesundheitsökonomische Beiträge, Bd. 54)

www.nomos-shop.de/22556

Portofreie Buch-Bestellungen unter www.nomos-shop.de Alle Preise inkl. Mehrwertsteuer

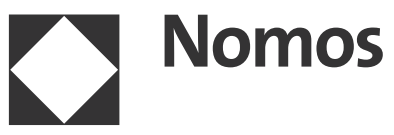

